\title{
Transcutaneous cervical root stimulation in the diagnosis of multifocal motor neuropathy with conduction block
}

\author{
R Arunachalam, A Osei-Lah, K R Mills
}

J Neurol Neurosurg Psychiatry 2003;74:1329-1331

Objectives: To determine if transcutaneous electrical stimulation of the cervical roots can be used to diagnose proximal conduction block (CB) in multifocal motor neuropathy (MMN) and whether it can reliably distinguish $M M N$ from amyotrophic lateral sclerosis (ALS).

Methods: Compound muscle action potentials (CMAPs) over the abductor digiti minimi (ADM) were evoked by supramaximal stimulation of the ulnar nerve at the wrist, below elbow, above elbow, axilla, Erb's point, and C8/T1 cervical roots in three groups of patients: 31 patients with ALS, nine patients with MMN, and 31 controls. Supramaximal stimulation at Erb's point and the $\mathrm{C} 8 / \mathrm{T} 1$ roots was carried out using a transcutaneous high voltage electrical stimulator. The negative peak amplitude, area, and duration of the CMAP were measured and normalised to that from the wrist. The percentage change in each segment in these parameters was calculated and compared between the different groups.

Results: At stimulation sites proximal to the elbow, there were no significant differences in relative CMAP amplitude, area, or duration between controls, ALS patients, and $M M N$ patients with clinically unaffected ulnar nerves. Similarly, the percentage segmental change between adjacent stimulation sites showed no significant differences. In six studies of MMN patients with weakness in ulnar hand muscles, the decrease in CMAP amplitude between the $C 8 / T 1$ roots and Erb's point exceeded the mean +2 SD of the control data.

Conclusion: Cervical root stimulation can quantify $C B$ in the most proximal segment of the ulnar nerve, a fall in CMAP amplitude if greater than $25 \%$, indicating block, and should be used routinely in the evaluation of patients suspected of having $M M N$, especially when distal stimulation has proved unhelpful.

M ultifocal motor neuropathy (MMN) classically presents as a chronic, progressive, asymmetrical, predominantly distal, upper limb weakness with variable degrees of atrophy and minimal or no sensory abnormalities. ${ }^{1-4}$

The clinical picture can mimic ALS and several cases of MMN have been initially misdiagnosed as ALS. ${ }^{56} \mathrm{MMN}$, however, has a better prognosis. ${ }^{7-9} \mathrm{~A}$ response to intravenous human immunoglobulin (ivIg) is reported in almost $80 \%{ }^{2}{ }^{10} \mathrm{It}$ is therefore critical to distinguish between the two conditions.

Conduction block (CB) is regarded as the electrophysiological hallmark of MMN and its detection is crucial in distinguishing MMN from ALS. ${ }^{51-13} \mathrm{CB}$ is defined by measuring the compound muscle action potential (CMAP) amplitude or area evoked by supramaximal nerve stimulation at adjacent sites. A CMAP reduction of $50 \%$ or more between adjacent sites is taken as clear evidence of $\mathrm{CB}$, provided the CMAP duration is not prolonged by more than $30 \% .{ }^{14}$ However, a cer- tain degree of CMAP reduction has been reported in ALS as a result of phase cancellation in the reduced number of surviving motor axons. ${ }^{15}{ }^{16}$ Computer modelling has shown that reductions in area of up to $50 \%$ can be caused by phase cancellation. ${ }^{17}$

Motor nerve conduction studies with stimulation up to the axilla will fail to detect $\mathrm{CB}$ occurring in the most proximal segments. It has been suggested that cervical root stimulation, either transcutaneously or by using a needle, be used to quantify CB in proximal segments. ${ }^{16} 1819$ Patients with ALS may have a greater degree of CMAP reduction in the proximal segments than in normal subjects because of abnormal temporal dispersion in fibres undergoing active degeneration. ${ }^{16}$ It is therefore important to determine the degree of CMAP reduction that can be found, not only in healthy controls but also in ALS.

\section{METHODS \\ Patients}

We studied four groups: patients with definite ALS by the El Escorial criteria $(\mathrm{n}=31,38$ sides), patients with MMN based on evidence of definite CB in two or more nerves and a clinical picture compatible with this diagnosis $(n=9,18$ sides), healthy control subjects $(n=17,26$ sides), and a group of patients with non-relevant neurological conditions $(n=14,16$ sides). One of the MMN patients was studied twice, two years apart, and one was studied three times at intervals of 1 year and 3 months. In all MMN patients ulnar nerves were studied bilaterally. If $\mathrm{CB}$ was found below Erb's point, then for the purpose of analysis, these data were excluded. Informed consent was obtained from all subjects.

\section{Stimulation and recording}

Motor conduction studies were carried out on the ulnar nerve with $\mathrm{Ag} / \mathrm{AgCl}$ recording electrodes using a belly-tendon montage on ADM. A conventional stimulator was used for stimulation up to the axilla. For proximal sites, stimulation was performed with a transcutaneous high voltage stimulator (Digitimer D180, Digitimer Ltd., Welwyn Garden City, UK). The cathode was placed over Erb's point with the anode $6 \mathrm{~cm}$ laterally. Cervical stimulation was applied with the cathode in the interspace between the C7 and Tl vertebral spines and the anode $6 \mathrm{~cm}$ laterally on the side being examined. Supramaximal stimulation was ensured by increasing the stimulus intensity and/or duration until no further increase in the CMAP was obtained. In the case of Erb's point and C8/T1 root stimulation, maximal CMAPs were usually obtained at stimulus intensities of $40-60 \%$ of the stimulator's maximal output (1500 V).

Abbreviations: $A D M$, abductor digiti minimi; $A L S$, amyotrophic lateral sclerosis; $C B$, conduction block; CMAP, compound muscle action potential; ivlg, intravenous immunoglobulin; $M M N$, multifocal motor neuropathy 
Table 1 The top panel displays the mean (SD) CMAP amplitude and change in duration normalised to the values from wrist stimulation in groups of patients with ALS, MMN with clinically unaffected ulnar nerves (MMN U), and controls. The bottom panel displays the mean (SD) percentage segmental change in CMAP amplitude and duration in the five segments studied in the ulnar nerve in the same groups of patients.

\begin{tabular}{|c|c|c|c|c|c|}
\hline & \multicolumn{5}{|c|}{ Mean (SD) CMAP parameters normalised to those from the wrist } \\
\hline & Below elbow & Above elbow & Axilla & Erb's point & $\mathrm{C} 8 / \mathrm{Tl}$ root \\
\hline \multicolumn{6}{|c|}{ Amplitude (\%) } \\
\hline Controls & $95.6(4.2)$ & $91.9(6.9)$ & $86.7(7.9)$ & $78.9(6.7)$ & $72.9(9.4)$ \\
\hline ALS & $89.6(8.2)$ & $81.9(10.9)$ & $86.1(9.5)$ & 75.5 (13.4) & $66.9(13.5)$ \\
\hline MMN U & $94.5(5.4)$ & $92.5(11.5)$ & $86.1(12.4)$ & $77.6(12.9)$ & $72.0(19.7)$ \\
\hline \multicolumn{6}{|c|}{ Duration change (\%) } \\
\hline Controls & $3.0(6.2)$ & $4.5(8.4)$ & $4.7(9.3)$ & $9.7(11.9)$ & $15.4(13.7)$ \\
\hline ALS & $6.2(8.7)$ & $9.3(12.3)$ & $10.4(12.2)$ & $18.1(17.1)$ & $21.2(20.7)$ \\
\hline \multirow[t]{3}{*}{ MMN U } & $1.3(9.1)$ & $4.3(9.9)$ & $5.3(10.5)$ & $22.9(23.9)$ & $27.9(33.2)$ \\
\hline & \multicolumn{5}{|c|}{ Percentage segmental change (SD) in CMAP parameters } \\
\hline & Below elbow-wrist & Across elbow & Axilla- above elbow & Erb's point-axilla & C8/T1 root-Erb's point \\
\hline \multicolumn{6}{|c|}{ Amplitude (\%) } \\
\hline Controls & $4.4(4.2)$ & $4.0(4.1)$ & $5.6(6.5)$ & $8.8(6.1)$ & $7.7(7.9)$ \\
\hline ALS & $10.4(8.2)$ & $3.9(6.9)$ & $4.6(9.2)$ & 7.7 (11.0) & 11.0 (12.2) \\
\hline MMN U & $5.5(5.4)$ & $2.3(8.0)$ & $6.8(8.6)$ & $9.4(11.1)$ & 8.5 (13.0) \\
\hline \multicolumn{6}{|l|}{ Duration (\%) } \\
\hline Controls & $3.0(6.2)$ & $1.4(3.1)$ & $0.3(7.4)$ & $4.9(6.7)$ & $5.2(5.5)$ \\
\hline ALS & $6.2(8.7)$ & $2.8(7.8)$ & $1.3(7.1)$ & $7.1(10.9)$ & $2.8(11.1)$ \\
\hline MMN U & $1.3(9.1)$ & $5.1(3.1)$ & $1.0(5.0)$ & $16.2(15.3)$ & $3.1(9.2)$ \\
\hline
\end{tabular}

The negative peak amplitude, area, and duration of all CMAPs were measured. If the CMAP amplitude evoked by wrist stimulation was less than $1 \mathrm{mV}$ then the data were excluded. This was the case in four ALS patients. To compare values across subjects, CMAP parameters were normalised to the CMAP obtained from wrist stimulation. The percentage change in each segment for all parameters was also calculated.

\section{Statistical analysis}

Unpaired two-tailed $t$ tests were used to assess significance of differences between the CMAP parameters of the different patient groups. A p value of $<0.05$ was considered significant.

\section{RESULTS}

The mean (SD) age of the neurological control group was 47.2 (13.8), that of the healthy control group was 36.4 (6.8), that of the ALS group was 57.3 (12.9), and that of the MMN group was 48.7 (13.7).

Comparison of the neurological control group and the healthy control group revealed no statistically significant differences and consequently the two groups were combined.
Comparing the combined control group with the ALS group (table 1), relative CMAP amplitude was significantly smaller in ALS below the elbow $(p=0.0001)$ and above the elbow $(p=0.003)$, but not at other stimulation sites. CMAP area from below the elbow was also significantly smaller $(p=0.01)$ in ALS, but this was not the case from above the elbow $(p=0.19)$. The small CMAP below the elbow in ALS was associated with an increase in relative CMAP duration $(p=0.06)$. No significant differences were found from other stimulation sites in CMAP amplitude $(p=0.11)$, CMAP area $(p=0.09)$ or CMAP duration $(p=0.12)$. The segmental change in CMAP amplitude and area (table 1) was significantly greater in ALS in the below elbow-wrist segment $(\mathrm{p}=0.001$ and $\mathrm{p}=0.01$ respectively) but no differences were found in other segments in amplitude, area, or duration.

Comparing the control group with the MMN group in whom no CB in the ulnar nerve was suspected (MMN-U, table 1), no significant differences were found in CMAP amplitude $(p=0.52)$, area $(p=0.39)$, or duration $(p=0.11)$ at any of the stimulation sites. Similarly, the segmental change in CMAP amplitude, area, and duration (table 1) were not significantly
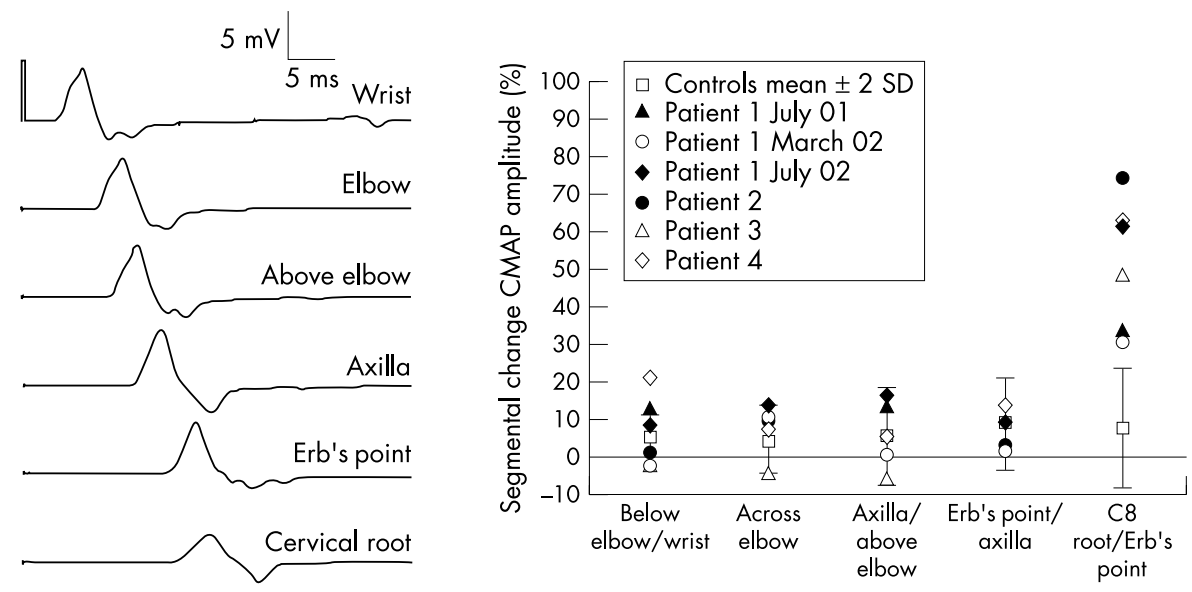

Figure 1 On the right, the segmental change in CMAP amplitude in the control group (mean \pm 2 SDs shown) and in 6 studies of MMN patients in whom there was clinical evidence of weakness in ulnar innervated hand muscles without $C B$ distal to Erb's point. On the left, CMAPs recorded from the ADM muscle in a patient with MMN from supramaximal stimulation of the ulnar nerve at the wrist, below the elbow, above the elbow, axilla, Erb's point and at the $\mathrm{C} 8 / \mathrm{T} 1$ root exit foramen. There is a significant decrease in CMAP amplitude and area without a significant increase in CMAP duration in the segment between the root exit and Erb's point. 
different in $\mathrm{MMN}(\mathrm{p}=0.52, \mathrm{p}=0.03$, and $\mathrm{p}=0.04$ respectively). No CB was detected in patients with clinically strong hands.

Of the 18 sides of patients with MMN, six showed no evidence of CB from Erb's point distally, but nevertheless had clinical weakness in ulnar innervated hand muscles. In two of these studies F-waves from ulnar stimulation at the wrist were absent. Data from these studies and an example of the original data from one patient are seen in fig 1 .

Considering the segment from the $\mathrm{C} 8 / \mathrm{Tl}$ roots to Erb's point, the mean (SD) percentage segmental change in CMAP amplitude in controls was 7.7 (7.9), CMAP area was 4.0 (9.6), and CMAP duration was 5.2 (5.5). Taking two standard deviations above the mean as a cut off for abnormality, the maximum percentage fall in this segment for CMAP amplitude is $23.4 \%$, for CMAP area is $23.2 \%$, and the percentage increase in duration is $16.2 \%$. In the six studies in patients with MMN in whom we found no evidence for $\mathrm{CB}$ in the ulnar nerve from Erb's point distally, the amplitude decrease exceeded $30 \%$ (fig 1 ), the area exceeded $25 \%$ and the duration increase did not exceed $15 \%$.

\section{DISCUSSION}

MMN is unusual among peripheral neuropathies in that focal abnormalities do not occur at the usual entrapment sites but tend to be located in the mid forearm, upper arm or, as we have shown here, in the motor roots. The most important differential diagnosis of MMN is ALS and clearly it is important to establish that changes in CMAP parameters in that condition could not be construed as conduction block. We have shown that the changes in CMAP parameters in ALS mirror those seen in control subjects, as do the parameters in MMN patients in whom CB is not suspected in ulnar nerve fibres. We therefore contend that proximal stimulation does not give rise to false positive diagnoses of CB in patients with ALS.

In MMN patients in whom CB was suspected but in whom no neurophysiological evidence of CB distal to Erb's point was found, we have shown that the segmental decrease in CMAP amplitude and area exceeded the mean +2 SDs of that seen in normals. We suggest that this is evidence of proximal CB in these patients. CB is suspected clinically if there is weakness without wasting of a muscle; neurophysiologically, absence of an F-response is also suggestive of CB. However, this cannot be relied upon as secure evidence of CB: in four of six ulnar nerves we studied which showed proximal block, there were preserved F-waves. Furthermore, the method described here is used to quantify the degree of $\mathrm{CB}$, which may be useful in tracking response to therapy.

A number of technical issues should be addressed. First, it is mandatory to establish that root stimulation is indeed supramaximal by progressively increasing intensity. However, if stimulus intensity is increased well beyond that required for a maximal CMAP there is a risk, especially in MMN, that stimulus current will spread beyond the block. This can be recognised by a sudden increase in CMAP amplitude and a shortening of latency as stimulus intensity is increased. It should also be noted that while magnetic stimulation over the cervical spine can also be used, a maximal CMAP cannot be guaranteed, especially in thick set individuals. ${ }^{20}$ Thus magnetic stimulation of cervical roots should not be used to quantify CB. Secondly, the choice of muscle is important. ADM occupies a privileged position in that it is relatively immune to volume conducted responses from other hand muscles. The same is not true of abductor pollicis brevis where proximal stimulation not uncommonly produces CMAPs larger than those evoked from distal sites because of volume conducted responses from nearby ulnar hand muscles. The technique therefore is not applicable for median nerve studies.

Needle stimulation of cervical roots has been reported, ${ }^{1621}$ but this technique has not been used systematically in MMN. The present technique is less invasive and well tolerated by patients. Usually we have been able to achieve CMAPs from root stimulation with-at most-five stimuli of increasing intensity.

We conclude that cervical root stimulation can be used to quantify $\mathrm{CB}$ in the most proximal segment of the ulnar nerve and should be used routinely in the evaluation of patients suspected of having MMN, especially when distal stimulation has proved unhelpful.

\section{ACKNOWLEDGEMENT}

Dr Osei-Lah and Professor Mills are grateful for the financial support they received from the Wellcome Trust.

\section{Authors' affiliations}

R Arunachalam, A Osei-Lah, K R Mills, Academic Neurosciences Centre, King's College Hospital, London, UK

Correspondence to: Professor Mills, Academic Neurosciences Centre, King's College Hospital, Denmark Hill, London SE5 9RS, UK; K.Mills@iop.kcl.ac.uk

\section{REFERENCES}

1 Hughes RAC. 79(th) ENMC International Workshop: multifocal motor neuropathy. 14-15 April 2000, Hilversum, The Netherlands. Neuromuscul Disord 2001;11:309-14

2 Nobile-Orazio E. Multifocal motor neuropathy. J Neuroimmunol 2001;115:4-18.

3 Chad DA, Hammer K, Sargent J. Slow resolution of multifocal weakness and fasciculation: a reversible motor neuron syndrome. Neurology 1986;36:1260-3

4 Parry GJ, Clarke S. Pure motor neuropathy with multifocal conduction block masquerading as motor neuron disease. Muscle Nerve 1985;8:167 (Abstract).

5 Bouche P, Moulonguet A, Younes-Chennoufi AB, et al. Multifocal motor neuropathy with conduction block: a study of 24 patients. J Neurol Neurosurg Psychiatry 1995;59:38-44.

6 Lange DJ, Trojaborg W, Latov N, et al. Multifocal motor neuropathy with conduction block: is it a distinct clinical entity? [see comments]. Neurology 1992;42:497-505

7 Ellis CM, Leary S, Payan J, et al. Use of human intravenous immunoglobulin in lower motor neuron syndromes. J Neurol Neurosurg Psychiatry 1999:67:15-9.

8 Bentes C, de Carvalho M, Evangelista T, et al. Multifocal motor neuropathy mimicking motor neuron disease: nine cases. J Neurol Sci 1999; 169:76-9.

9 Molinuevo JL, Cruz-Martinez A, Graus F, et al. Central motor conduction time in patients with multifocal motor conduction block. Muscle Nerve 1999;22:926-32.

10 Van den Berg LH, Kerkhoff H, Oey PL, et al. Treatment of multifocal motor neuropathy with high dose intravenous immunuglobins: a double blind placebo controlled study. J Neurol Neurosurg Psychiatry 1995:59:248-52.

11 Parry GJ, Clarke S. Multifocal acquired demyelinating neuropathy masquerading as motor neuron disease. Muscle Nerve 1988;11:103-7.

12 Chaudhry V, Corse AM, Cornblath D, et al. Multifocal motor neuropathy: electrodiagnostic features. Muscle Nerve 1994;17:198205.

13 Comi G, Amadio S, Galardi G, et al. Clinical and neurophysiological assessment of immunoglobulin therapy in five patients with multifocal motor neuropathy. J Neurol Neurosurg Psychiatry 1994;57:35-7.

14 Olney RK. Consensus criteria for the diagnosis of partial conduction block. Muscle Nerve 1999;22 (Suppl):S225-9.

15 Katz JS, Wolfe GI, Bryan WW, et al. Electrophysiologic findings in multifocal motor neuropathy. Neurology 1997;48:700-7.

16 Raynor EM, Shefner JM, Ross M, et al. Root stimulation studies in the evaluation of patients with motor neuron disease. Neurology 1998;50: 1907-9.

17 Rhee EK, England JD, Sumner AJ. A computer simulation of conduction block: effects produced by actual versus interphase cancellation. Ann Neurol 1990;28:146-56.

18 Ormerod IE, Waddy HM, Kermode AG, et al. Involvement of the central nervous system in chronic inflammatory demyelinating polyneuropathy: a clinical, electrophysiological and magnetic resonance imaging study [comment]. J Neurol Neurosurg Psychiatry 1990;53:789-93.

19 Mills KR, Murray NM. Proximal conduction block in early Guillain-Barre syndrome. Lancet 1985;2:659.

20 Mills KR, Murray, NM. Electrical stimulation over the human vertebral column: which neural elements are excited? Electroencephalogr Clin Neurophysiol 1986;63:582-9

21 Berger AR, Busis NA, Logigian EL, et al. Cervical root stimulation in the diagnosis of radiculopathy. Neurology 1987;37:329-32. 